Chemical Physics 132 (1989) 71-81

North-Holland, Amsterdam

\title{
THE FLUID MECHANICS OF PHOTOTHERMAL SYSTEMS
}

\author{
S.J. JACOBS \\ Department of Atmospheric, Oceanic, and Space Sciences, Department of Mechanical Engineering and Applied Mechanics, \\ The University of Michigan, Ann Arbor, MI 48109, USA
}

Received 25 April 1988

\begin{abstract}
The flow of a binary mixture of perfect gases confined in a cylindrical container and excited by a pulsed laser beam is treated using linear theory. Chebyshev methods and a variable time step implicit scheme are employed to obtain an accurate numerical method for solving the equations of motion. The results of sample calculations are presented to assess previous theories of the flow in photothermal systems.
\end{abstract}

\section{Introduction}

As noted by Barker and Toselli [1], photothermal methods provide a reliable method for measuring material properties, energy transfer rates, and chemical reaction rates in fluids. Examples of the application of such techniques are the time-resolved thermal lensing experiments of Grabiner et al. [2] and the time-resolved optoacoustics studies of Presser et al. [3]. In these and other studies, the interpretation of the data depends on the availability of a theoretical solution of the governing equations of fluid motion.

Although nonlinear effects are undoubtedly important in some recent experiments, such as those of Guckert and Carr [4], for most cases of interest the flow can be analyzed using a linear theory. Previous treatments along these lines can be grouped in three categories, the acoustic wave theories of Siebert [5] and Bailey et al. [6], in which diffusive effects are neglected, a study by Cruickshank and co-workers [7], in which diffusive effects are included but acoustic effects are ignored by omitting the material derivative of the pressure in the energy equation of fluid mechanics, and a combined theory given in its original form by Barker and Rothem [8] and revised in ref. [1]. Effects due to the finite extent of the fluid are considered in the papers by Barker and co-workers but are ignored for the most part in the other treatments.

Refs. [5-7] are based on the argument that the time scale for the decay of acoustic waves is short compared to the diffusive time scale, so that diffusion can be neglected in treating the initial phase of the motion and acoustic effects in solving the governing equations for larger times. The drawbacks of this approach are the slow decay of cylindrical acoustic waves [9], which implies that the scaling argument is oversimplified, the difficulty in determining initial conditions for treating the flow after the decay of the waves, and the problem of estimating when effects due to the finite volume of the container invalidate the approximate solution for the diffusive phase of the flow. A theoretical solution in which acoustic, diffusive, and finite volume effects are included can be obtained using the separation of variables technique of Barker and co-workers. However, in both the original and revised versions of the theory viscosity is neglected in an inconsistent approximation and the boundary condition of vanishing normal component of velocity at a solid boundary is not satisfied. Therefore, despite the considerable progress which has been made in analyzing the problem, it remains to be shown that previous treatments of the fluid mechanics of photothermal systems provide theoretical solutions with an accuracy comparable to that of the experiments.

The purpose of the present study is to remedy this situation by providing a reliable numerical scheme for treating the combined effects of compressibility, diffusive and viscous effects, and energy transfer on flows 
confined in a cylindrical container. Our aims in working out such a method are to give a mcans for testing the theories discussed above and a procedure for the analysis of experimental data if these treatments prove to be inadequate. In addition, although our work is based on a linear theory, it can be generalized in a straightforward manner to provide a method for treating strongly nonlinear flows of the type encountered in the experiments of Guckert and Carr.

In the next section the governing equations are linearized and expressed in dimensionless form. This is followed by an outline of the numerical scheme and a presentation of the results of a sample calculation.

\section{Formulation}

Let $m_{i}$ denote the molecular mass of species $i(i=1,2)$, where both species are treated as ideal gases, and let $h_{i}$ denote the enthalpy per molecule of species $i, C_{v}$ and $k$ the specific heat at constant volume of the mixture and the Boltzmann constant, $\boldsymbol{u}$ the barycentric velocity, $c$ the mass fraction of species $1, p$ the pressure, $\rho$ the density, and $T$ the temperature. We also define $q$ as the heat flux vector, $i$ and $K$ as the diffusive mass flux vector and reaction rate for species $1, \tau$ as the viscous stress tensor, and $\mathrm{D} / \mathrm{D} t$ as the material derivative. Then, following Landau and Lifshitz [9] and Hirschfelder et al. [10], and neglecting body forces and the Soret and Dufour effects, we obtain the governing equations in the form

$p=\left[c / m_{1}+(1-c) / m_{2}\right] k \rho T$,

$\frac{\mathrm{D} \rho}{\mathrm{D} t}+\rho \nabla \cdot \boldsymbol{u}=0$,

$\rho \frac{\mathrm{D} c}{\mathrm{D} t}=K-\nabla \cdot i$

$\rho \frac{\mathrm{D} u}{\mathrm{D} t}+\nabla p=\nabla \cdot \tau$

and

$\rho C_{v} \frac{\mathrm{D} T}{\mathrm{D} t}+p \nabla \cdot \boldsymbol{u}=\tau: \nabla \boldsymbol{u}-\boldsymbol{\nabla} \cdot \boldsymbol{q}+\bar{u}(\boldsymbol{\nabla} \cdot \boldsymbol{i}-K)$,

where $\bar{u}$ and a related enthalpy $h$ are defined by

$\bar{u}=\left(h_{1}-k T\right) / m_{1}-\left(h_{2}-k T\right) / m_{2}, \quad h=h_{1} / m_{1}-h_{2} / m_{2}$.

Letting I denote the unit tensor, the constitutive equations are

$\boldsymbol{\tau}=\mu\left[(\boldsymbol{\nabla} \boldsymbol{u})+(\boldsymbol{\nabla} \boldsymbol{u})^{\mathrm{T}}\right]+\left(\zeta-\frac{2}{3} \mu\right)(\nabla \cdot \boldsymbol{u}) \boldsymbol{l}$,

$\boldsymbol{q}=h \boldsymbol{i}-\kappa \nabla T, \quad i=-\rho D\left[\nabla c+\left(k_{p} / p\right) \nabla p\right]$,

in which $\mu$ is the shear viscosity, $\zeta$ is the second viscosity, $\kappa$ is the thermal conductivity, $D$ is the binary diffusion coefficient, and

$k_{p}=\left(m_{2}-m_{1}\right) c(1-c)\left[c / m_{1}+(1-c) / m_{2}\right]$

is the ratio of the barodiffusion coefficient to $D$. The boundary conditions at a solid stationary boundary with normal $\hat{\boldsymbol{n}}$ are $\boldsymbol{u}=\mathbf{0}$, either $c=0$ or $\hat{\boldsymbol{n}} \cdot \boldsymbol{i}=0$, depending on the model, and $T$ constant, assuming that the boundary remains at its initial temperature.

We consider here a fluid initially at rest with concentration $c=0$ for which instantaneous changes in the con- 
centration and temperature are induced by the passage of a pulsed laser beam. Assuming that the density and the velocity are unchanged by the action of the beam, letting $\rho_{0}$ denote the initial value of $\rho$, and defining $f_{+}$as the value of any variable $f$ at $t=0$, immediately after the passage of the beam, the initial conditions are

$\rho=\rho_{0}, \quad c=c_{+}, \quad T=T_{+}, \quad \boldsymbol{u}=\mathbf{0}$,

at $t=0$, where $c_{+}$and $T_{+}$are functions of position. In addition, we restrict our attention to axially symmetric flow in a cylinder whose height is large compared to the thickness of the boundary layers at the ends of the cylinder. To lowest order in a boundary layer approximation, variation of the dependent variables with distance along the cylinder axis can be neglected in the main body of the fluid, as can the flow due to displacement thickness, and the velocity in this region is entirely radial. Consequently, letting $u_{r}$ denote the radial component of velocity, eqs. (1)-(5) together with the boundary and initial conditions determine $p, \rho, C, T$, and $u_{r}$, all of which depend only on radius $r$ and time $t$ in the main body of the fluid.

Assuming that perturbations to the initial state are small, the governing equations can be linearized by introducing the perturbation quantities

$p^{\prime}=p-p_{0}, \quad \rho^{\prime}=\rho-\rho_{\rho}, \quad T^{\prime}=T-T_{0}$,

where $p_{0}$ and $T_{0}$ arc the initial pressure and temperature, and by neglecting terms nonlinear in $\boldsymbol{\mu}, c$, and the primed variables. Taking $K$ as

$K=-\rho_{0} k c$

and noting that barodiffusion and variations of the scalars in the constitutive equations can be neglected in a linearized theory, the linearized form of the governing equations is given by

$\frac{p^{\prime}}{p_{0}}=\frac{\rho^{\prime}}{\rho_{0}}+\frac{T^{\prime}}{T_{0}}+\left(\frac{m_{2}-m_{1}}{m_{1}}\right) c$

$\frac{\partial}{\partial t}\left(\frac{\rho^{\prime}}{\rho_{0}}\right)=-\left(\frac{\partial u_{r}}{\partial r}+\frac{u_{r}}{r}\right)$

$\frac{\partial c}{\partial t}=D \nabla^{2} c-k c$

$\frac{\partial u_{r}}{\partial t}=-\frac{p_{0}}{\rho_{0}} \frac{\partial}{\partial r}\left(\frac{p^{\prime}}{p_{0}}\right)+\left(\frac{4 \mu / 3+\zeta}{\rho_{0}}\right)\left(\nabla^{2} u_{r}-\frac{u_{r}}{r^{2}}\right)$

and

$\frac{\partial}{\partial t}\left(\frac{T^{\prime}}{T_{0}}\right)=(\gamma-1)\left[\left(\frac{m_{2}-m_{1}}{m_{1}}\right)\left(D \nabla^{2} c-k c\right)-\frac{\partial u_{r}}{\partial r}-\frac{u_{r}}{r}\right]+\gamma\left[\frac{\kappa}{\rho_{0} C_{p}} \nabla^{2}\left(\frac{T}{T_{0}}\right)+\frac{k \bar{h}}{C_{p} T_{0}} c\right]$,

where $C_{p}$ is the specific heat at constant pressure, $\gamma=C_{p} / C_{v}$, and

$\nabla^{2}=\frac{\partial^{2}}{\partial t^{2}}+\frac{1}{r} \frac{\partial}{\partial r}$

is the Laplacian operator in polar coordinates for axisymmetric flow. The boundary conditions are $u_{r}=0$ at $r=L$, the outer boundary of the cylinder, $c=0$ or $\partial c / \partial r=0$ at $r=L, T^{\prime}=0$ at $r=L$, and all dependent variables finite at $r=0$. The initial conditions are obtained by combining (10) and (11).

Substituting the expression for $p^{\prime} / p_{0}$ from (13) into (16) provides a set of four evolution equations for $\rho^{\prime}$, $T^{\prime}, c$, and $u_{r}$, which we now express in dimensionless form. Let $V$ denote the speed of sound evaluated for the initial state, $c_{\mathrm{m}}$ the maximum value of the concentration at $t=0$, and 
$A=\left(\bar{h} c_{\mathrm{m}} / C_{p} T_{0}\right) k L / V$

where, according to linear theory, $C_{p}$ and $\bar{h}$ are evaluated at the initial thermodynamic state. Hence, introducing dimensionless variables through

$x_{*}=-1+2(r / L)^{2}, \quad t_{*}=V t / L, \quad U_{*}=L u_{r} / A V r$,

$C_{*}=c / c_{\mathrm{m}}, \quad \theta_{*}=T^{\prime} / A T_{0}, \quad \sigma_{*}=\rho^{\prime} / A \rho_{0}$,

and omitting the asterisks, we obtain

$\frac{\partial \sigma}{\partial t}=-2\left((1+x) \frac{\partial U}{\partial x}+U\right)$

$\frac{\partial C}{\partial t}=8 k_{1}\left((1+x) \frac{\partial^{2} C}{\partial x^{2}}+\frac{\partial C}{\partial x}\right)-k_{2} C$

$\frac{\partial U}{\partial t}=-\frac{4}{\gamma} \frac{\partial}{\partial x}\left(\sigma+\theta+k_{5} C\right)+8 k_{3}\left((1+x) \frac{\partial^{2} U}{\partial x^{2}}+2 \frac{\partial U}{\partial x}\right)$

and

$$
\begin{aligned}
\frac{\partial \theta}{\partial t} & =(\gamma-1)\left[8 k_{1} k_{5}\left((1+x) \frac{\partial^{2} C}{\partial x^{2}}+\frac{\partial C}{\partial x}\right)-2\left((1+x) \frac{\partial U}{\partial x}+U\right)\right] \\
& +8 k_{4} \gamma\left((1+x) \frac{\partial^{2} \theta}{\partial x^{2}}+\frac{\partial \theta}{\partial x}\right)+\left[\gamma-k_{2} k_{5}(\gamma-1)\right] C .
\end{aligned}
$$

The boundary and initial conditions are

$U=0, \quad C=0$ or $\partial C / \partial x=0, \quad \theta=0$,

at $x=1$, the outer boundary of the cylinder, all dependent variables finite at $x=-1$, the axis of the cylinder, and

$\sigma=0, \quad U=0, \quad C=C_{+}(x), \quad \theta=\theta_{+}(x)$,

at $t=0$, where $C_{+}$and $\theta_{+}$are prescribed functions of $x$. Here the $k_{i}$ are dimensionless parameters defined by

$k_{1}=D / L V, \quad k_{2}=k L / V, \quad k_{3}=(4 \mu / 3+\zeta) / \rho_{0} L V, \quad k_{4}=\kappa / \rho_{0} C_{p} L V$,

$k_{5}=\left(C_{p} T_{0} / \bar{h}\right)(V / k L)\left(m_{2}-m_{1}\right) / m_{1}$,

and $A$ is a measure of nonlinear effects. The present calculation is based on the assumption that $A \ll 1$.

The above partial differential equations admit a solution of the form

$(C, \sigma, \theta)=\left(c_{1}(t), c_{2}(t), c_{3}(t)\right) J_{0}(\lambda R), \quad U=c_{4}(t) J_{1}(\lambda R) / R$,

where $R=r / L$ is the dimensionless radius, $J_{\nu}$ is a Bessel function, $\lambda$ is a solution of $J_{1}(\lambda)=0$ if $U, \partial \theta / \partial x$, and $\partial C / \partial x$ vanish at the boundary of the cylinder, and $\lambda$ solves $J_{0}(\lambda)=0$ if $\partial U / \partial x, \theta$, and $C$ vanish at the boundary. The resulting ordinary differential equations for the $c_{i}$ can be solved without difficulty, and the solution can be generalized through use of Fourier-Bessel series. However, an analytical solution is much more difficult to obtain if the physical boundary conditions (25) are imposed and is unavailable if nonlinear effects are significant or if the rate parameter $k_{2}$ varies with time, and so, in general, the governing equations must be solved numerically. 


\section{Numerical solution}

In the present study the problem is analyzed using Chebyshev methods, as described by Orszag and co-workers in refs. $[11,12]$. These are based on the approximation of functions $f(x)$ defined in the region $x \in[-1,1]$ by expansions of the form

$f(x)=\sum_{n=0}^{M} A_{n} T_{n}(x)$,

where the Chebyshev polynomials $T_{n}(x)$ are polynomials of order $n$ defined in the same region by

$T_{n}(x)=\cos \left(n \cos ^{-1} x\right), \quad n=0,1, \ldots$.

As noted in the numerical analysis literature, Chebyshev series converge rapidly, and consequently provide an exceptionally accurate approximation if $f$ is sufficiently smooth.

The series (29) can be summed numerically using the recurrence relation

$B_{M+2}=B_{M+1}=0$, for $j=M$ down to $0 \quad B_{j}=2 x B_{j}-B_{j+2}+A_{j}$,

which yields $f(x)=B_{0}-x B_{1}$. Conversely, the coefficients $A_{n}$ are calculated in terms of $f_{j+1}$ by defining $c_{n}$ through $c_{0}=c_{M}=2, c_{n}=1(1<n<M)$, and grid points $x_{n}$ by $x_{n}=\cos (\pi n / M),(0 \leqslant n \leqslant M)$. The inverse recurrence relation is

$B_{M+2}=B_{M+1}=0, \quad$ for $j=M$ down to $0 \quad B_{j}=2 x_{n} B_{j+1}-B_{j+2}+f\left(x_{j}\right) / c_{j}$,

and $A_{n}$ is given by

$A_{n}=\frac{2}{M c_{n}}\left(B_{0}-x_{n} B_{1}\right)$.

The function $f$ and its Chebyshev coefficients also satisfy

$f\left(x_{n}\right)=\sum_{j=0}^{M} A_{j} \cos (\pi j n / M)$,

where

$A_{n}=\frac{2}{M c_{n}} \sum_{j=0}^{M} \frac{f\left(x_{j}\right)}{c_{j}} \cos (\pi j n / M)$,

in which both sums can be evaluated using fast Fourier transform methods.

As shown in ref. [12], derivatives of $f$ at the grid points $x_{n}$ are given by

$f^{\prime}\left(x_{n}\right)=\sum_{k=0}^{M} P_{n k} f\left(x_{k}\right), f^{\prime \prime}\left(x_{n}\right)=\sum_{k=0}^{M} Q_{n k} f\left(x_{k}\right)$,

in which

$P_{00}=-P_{M M}=\frac{2 M^{2}+1}{6}, \quad P_{n n}=\frac{x_{n}}{2\left(x_{n}^{2}-1\right)} \quad(n>0$ and $n<M)$,

$P_{n k}=\frac{c_{n}}{c_{k}} \frac{(-1)^{n+k}}{x_{n}-x_{k}} \quad(n \neq k)$,

and where the matrix $\mathbf{Q}=\mathbf{P P}$. Similarly, recurrence relations given in ref. [11] can be used to calculate the Chebyshev coefficients of $f^{\prime}$ and $f^{\prime \prime}$ in terms of the coefficients of $f$. 
The most commonly used Chebyshev methods are pseudospectral collocation, which consists of satisfying the field equations at the grid points $x_{n}$ and of approximating spatial derivatives through use of (36), and the spectral tau method, in which the governing equations are solved for the Chebyshev coefficients and in which spatial derivatives are calculated using the recurrence relations given in ref. [11]. Pseudospectral collocation is better adapted for treating nonlinear effects, and therefore only this method will be described in detail. Although the computing strategy is different, the corresponding equations for the spectral tau method are virtually identical.

Pseudospectral collocation is implemented here by applying (21) at the points $x_{n}$ for $n=0, \ldots, M$, and (22)(24) at the points $x_{n t}$ for $n=1, \ldots, M$. The boundary conditions are satisfied by noting that

$f\left(x_{0}\right)=0$ or $f\left(x_{0}\right)=-\sum_{k=1}^{M} \frac{P_{0 k}}{P_{00}} f\left(x_{k}\right)$,

where the first equation holds if $f(1)=0$ and the second if $f^{\prime}(1)=0$, and so, defining

$r_{k}=0, \quad$ if $f(1)=0 ; \quad r_{k}=P_{0 k} / P_{00}, \quad$ if $f^{\prime}(1)=0$,

we find that

$\sum_{k=0}^{M} L_{n k} f\left(x_{k}\right)=\sum_{k=1}^{M}\left[L_{n k}-L_{n_{0}} r_{k}\right] f\left(x_{k}\right)$,

if $f(1)=0$ or $f^{\prime}(1)=0$, where $\mathbf{L}$ is any matrix. Analogous relations hold for sums involving the Chebyshev coefficients if $f$ or $f^{\prime}$ vanishes at $x=1$.

To express the collocation equations for the present problem, we define $f_{n}=f\left(x_{n}\right)$ for any function $f(x)$ and the matrices $R_{n k}$ and $S_{n k}$ by

$R_{n k}=8\left[\left(1+x_{n}\right) Q_{n k}+P_{n k}\right], \quad S_{n k}=8\left[\left(1+x_{n}\right) Q_{n k}+2 P_{n k}\right]$,

and apply the boundary conditions using (40) to obtain the set of $4 M+1$ ordinary differential equations

$\frac{\mathrm{d} \sigma_{0}}{\mathrm{~d} t}=-4 \sum_{k} P_{0 k} U_{k}$

and

$\frac{\mathrm{d} \sigma_{n}}{\mathrm{~d} t}=-2\left(1+x_{n}\right) \sum_{k} P_{n k} U_{k}-2 U_{n}$,

$\frac{\mathrm{d} C_{n}}{\mathrm{~d} t}=k_{1} \sum_{k}\left[R_{n k}-R_{n 0} p_{k}\right] C_{k}-k_{2} C_{n}$,

$\frac{\mathrm{d} U_{n}}{\mathrm{~d} t}=k_{3} \sum_{k} S_{n k} U_{k}-\frac{4}{\gamma}\left(P_{n 0} \sigma_{0}+\sum_{k} P_{n k} \sigma_{k}+\sum_{k}\left[P_{n k}-P_{n 0} r_{k}\right] \theta_{k}+k_{5} \sum_{k}\left[P_{n k}-P_{n 0} p_{k}\right] C_{k}\right)$,

$\frac{\mathrm{d} \theta_{n}}{\mathrm{~d} t}=-2(\gamma-1)\left(\left(1+x_{n}\right) \sum_{k} P_{n k} U_{k}+U_{n}\right)+\left[\gamma-k_{2} k_{5}(\gamma-1)\right] C_{n}$

$+\sum_{k}\left\{k_{1} k_{5}(\gamma-1)\left[R_{n k}-R_{n 0} p_{k}\right] C_{k}+k_{4} \gamma\left[R_{n k}-R_{n 0} r_{k}\right] \theta_{k}\right\}$,

where (43)-(46) hold for $1 \leqslant n \leqslant M$ and where the sums run over $k$ from 1 to $M$. Here $p_{k}$ is defined by the first relation in (39) or the second, depending on the boundary condition on $C$, and $r_{k}$ is defined by the first relation in (39) if $\theta=0$ at $x=1$ and by the second if $\partial \theta / \partial x=0$ at $x=1$. These equations must be solved subject to the initial conditions

$\sigma_{n}(0)=0, \quad U_{n}(0)=0, \quad C_{n}(0)=C_{+}\left(x_{n}\right), \quad \theta_{n}(0)=\theta_{+}\left(x_{n}\right)$. 
In most fluid dynamics computations involving Chebyshev methods the solution is advanced in time using a low-order implicit procedure, such as the trapezoidal rule. In our opinion this is a mistake, since both low- and high-order implicit methods require one LU decomposition per time step, and the cost of the additional backsolves needed to implement a higher-order method is comparatively low. Accordingly, in the present study we employ such a scheme, a fourth-order variable step Wanner-Rosenbrock method in the form described by Shampine [13] and Gottwald and Wanner [14]. As in the more familiar Runge-Kutta-Fehlberg method, which cannot be employed here because the ordinary differential equations which must be solved in the present study are unequivocally stiff, Wanner-Rosenbrock methods compute numerical solutions of different orders of accuracy and use the difference between these solutions for step size control.

In the version of the method used here, the set of ordinary differential equations

$\frac{\mathrm{d} u}{\mathrm{~d} t}=f(t, \boldsymbol{u}), \quad \boldsymbol{u}=\boldsymbol{u}_{0} \quad$ at $t=t_{0}$,

is solved numerically to obtain

$\boldsymbol{u}\left(t_{0}+h\right)=\boldsymbol{u}\left(t_{0}\right)+h \sum_{i=1}^{4} m_{i} \boldsymbol{k}_{i}$

where the $\boldsymbol{k}_{i}$ satisfy

$\mathbf{E} \boldsymbol{k}_{i}=f\left(t_{0}+A_{i} h, \boldsymbol{u}_{0}+\sum_{k=1}^{i-1} a_{i k} \boldsymbol{k}_{k}\right)+B_{i} h \boldsymbol{d}+\sum_{k=1}^{i-1} c_{i k} \boldsymbol{k}_{k}$,

for $i=1, \ldots, 4$. Here

$\mathrm{J}=\nabla_{u} f, \quad \mathrm{E}=\mathrm{I}-\Gamma h \mathrm{~J}, \quad \boldsymbol{d}=\frac{\partial f}{\partial t}$,

where $\mathbf{J}$ and $\boldsymbol{d}$ are evaluated at $(t, \boldsymbol{u})=\left(t_{0}, \boldsymbol{u}_{0}\right), \mathrm{J}$ is the Jacobian matrix, $\mathbf{I}$ is the identity matrix, $h$ is the step size, $\Gamma, A_{i}, a_{i k}, B_{i}, c_{i k}$, and $m_{i}$ are constants, and, by an appropriate choice of the $m_{i}(49)$ provides a solution of either third-order or fourth-order accuracy.

In Shampine's version of the method, $\Gamma=1 / 2$ and the other constants are chosen to yield the system

$$
\begin{aligned}
& E \boldsymbol{k}_{1}=f\left(t_{0}, \boldsymbol{u}_{0}\right)+\frac{1}{2} h \boldsymbol{d}, \\
& \mathrm{E} \boldsymbol{k}_{2}=\boldsymbol{f}\left(t_{0}+h, \boldsymbol{u}_{0}+h \boldsymbol{k}_{1}\right)-\frac{3}{2} h \boldsymbol{d}-4 \boldsymbol{k}_{1}, \\
& \mathrm{E} \boldsymbol{k}_{3}=\boldsymbol{f}\left(t_{0}+\frac{3}{5} h, \boldsymbol{u}_{0}+\frac{24}{25} h \boldsymbol{k}_{1}+\frac{3}{25} h \boldsymbol{k}_{2}\right)+\frac{121}{50} h d+\frac{186}{25} \boldsymbol{k}_{1}+\frac{6}{5} \boldsymbol{k}_{2}, \\
& \mathrm{E} \boldsymbol{k}_{4}=f\left(t_{0}+\frac{3}{5} h, \boldsymbol{u}_{0}+\frac{24}{25} h \boldsymbol{k}_{1}+\frac{3}{25} h \boldsymbol{k}_{2}\right)+\frac{29}{250} h d-\frac{56}{125} \boldsymbol{k}_{1}-\frac{27}{125} \boldsymbol{k}_{2}-\frac{1}{5} \boldsymbol{k}_{3},
\end{aligned}
$$

from which it follows that

$\boldsymbol{u}_{4}\left(t_{0}+h\right)=\boldsymbol{u}_{0}+h\left(\frac{19}{18} \boldsymbol{k}_{1}+\frac{1}{4} \boldsymbol{k}_{2}+\frac{25}{216} \boldsymbol{k}_{3}+\frac{125}{216} \boldsymbol{k}_{4}\right)$,

and

$D=h\left(\frac{17}{108} k_{1}+\frac{7}{72} k_{2}+\frac{125}{216} k_{4}\right)$.

In this set of equations, (53) provides a solution for $u$ at time $\left(t_{0}+h\right)$ with fourth-order accuracy, and (54) the difference between the third-order and fourth-order solutions needed for step size adjustment. A similar system of equations, which yields a more accurate but less stable numerical scheme, is given in the article by Gottwald and Wanner.

In treating the present problem it is convenient to use a working array of dimension $4 M+1$, for which $C_{k}, \theta_{k}$, 
$U_{k}$, and $\sigma_{k}(1 \leqslant k \leqslant M)$ are the first $4 M$ components, and $\sigma_{0}$ is the last component. Then, to solve an equation of the form $E k=u$, we use the partition

$k=[a, b, c, d], \quad u=[p, q, r, s]$,

where $\boldsymbol{d}$ and $\boldsymbol{s}$ have $M+1$ components and the other vectors have $M$ components, and express $\boldsymbol{E} \boldsymbol{k}=\boldsymbol{u}$ by

$\mathrm{B}_{1} a=p$,

$\mathrm{B}_{2} a+\mathrm{B}_{3} b+\mathrm{B}_{4} c=q$,

$\mathrm{B}_{5} a+\mathrm{B}_{6} b+\mathrm{B}_{7} c+\mathrm{B}_{8} d=r$,

$\mathrm{B}_{9} c+\mathrm{B}_{10} d=s$,

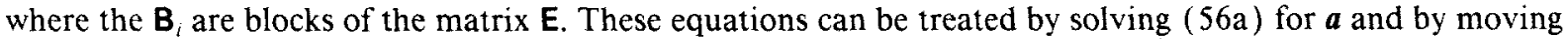
the terms involving $a$ to the right sides of $(56 \mathrm{~b})$ and (56c) to obtain a block tridiagonal system for the remaining unknowns. Hence, we replace an LU decomposition of the $(4 M+1) \times(4 M+1)$ matrix E by LU decompositions of four smaller matrices, each of dimension approximately one fourth of the dimension of $E$. Since carrying out an LU decomposition for an $N \times N$ matrix involves $O\left(N^{3}\right)$ multiplications and divisions, this procedure reduces the number of multiplications and divisions involved in the solution of $(52)$ by approximately a factor of sixteen.

The above analysis has been employed to write Pascal programs DIFCOLL and DIFCHEB, in which the first program is based on pscudospectral collocation and the second on the spectral tau method. In both programs the user prescribes the initial concentration and temperature profiles by defining Pascal functions before compilation. Prior to advancing the solution in time, the program interpolates and files the current solution at equally spaced increments in $R$. The current value of $\partial^{2} \sigma / \partial R^{2}$ on the cylinder axis, which is needed in applications, is also calculated and filed. The Rosenbrock-Wanner method is then invoked to obtain the solution at the next time step, using either Shampine's or Gottwald and Wanner's numerical scheme. The user specifies which scheme to use at run time, along with $M$, the number of grid points, TOL, the error tolerance used for step size control, and the initial and minimum temporal step sizes.

The step size control method used in DIFCOLL and DIFCHEB is similar to that of Gottwald and Wanner, but differs in that we allow the step size to increase by as much as a factor of five if the norm of the Jacobian matrix $\mathbf{J}$ is small, as suggested by Shampine, and in our decision to halve the step size each time a computed solution fails a tolerance test based on the magnitude of (54), again as suggested by Shampine. In order to determine the accuracy of the spatial representation, we use the notion that the spatial resolution is adequate if the highest-order Chebyshev coefficients are small. This check is implemented in DIFCOLL and DIFCHEB by computing the ratio of the average of the absolute values of the Chebyshev coefficients with the two highest indices to the absolute value of the coefficients with the lowest index at each time step. As another check on accuracy, the integral of $\sigma$ over the area of the cylinder, which should vanish for all $t \geqslant 0$ in order to conserve mass, is computed numerically at each time step.

To test the programs, numerical solutions were calculated using parameter values for experiments of the type conducted by Professor Barker and Dr. Toselli, and by treating the flow contained by an insulated boundary with initial conditions $\sigma=\theta=U=0, C=J_{0}(\lambda R)$, where $\lambda$ is the smallest zero of $J_{1}(\lambda)$. Computing the solution for $M=25$ using DIFCOLL and DIFCHEB and both Shampine's scheme and that of Gottwald and Wanner yields numerical solutions for $\sigma, \theta, C$, and $\partial^{2} \sigma / \partial R^{2}$ for $R=0, t=0.1$, which differ from each other and from the separation of variables solution outlined at the end of section 2 by fractional errors of order $5 \times 10^{-8}$ or smaller. In addition, the computed value of the integrated density perturbation $\sigma$ was found to vanish to machine accuracy.

As another test of the method, we note that for small values of $t$ the solution for initial concentration and temperature profiles strongly peaked at the cylinder axis is approximately the same as that for flow in an infinite 
fluid, and therefore can be obtained using Hankel transforms. In particular, if $C=\exp \left(-\lambda R^{2}\right)$ at $t=0$, the Hankel transform solution for $C$ is given by

$C=\frac{1}{1+4 \lambda k_{1} t} \exp \left(-\frac{\lambda R^{2}}{1+4 \lambda k_{1} t}-k_{2} t\right)$

which can be compared to the numerical solution. Tests using DIFCOLL and DIFCHEB for various large values of $\lambda$ for $t \leqslant 0.1$ show that the numerical solution for $C$ and (57) agree with errors of at most a few percent except very near the boundary of the cylinder. Accordingly, our view is that the numerical procedures used in DIFCOLL and DIFCHEB are correct in principle, and can yield extremely accurate results if enough grid points are employed.

As a more substantive test of the scheme, DIFCOLL was used in its Shampine version to treat a model proposed in ref. [8] for the excitation of $\mathrm{NO}_{2}$ with $\mathrm{Ar}$ as a buffer gas. In the calculations the initial values of $C$ and $\theta$ are given by $\exp \left(-\lambda R^{2}\right)$ and 0 , respectively, with $\lambda=156.25$, and it is assumed that $C=0$ on the boundary of the cylinder, $r=1.25 \mathrm{~cm}$ in dimensional units. In all runs the initial and minimum temporal step sizes were taken as $10^{-4}$ in dimensionless units. The other parameter values used in the numerical experiments are given in table 1 . In treating cases 1 and 2, the number of grid points needed for the calculation was determined by carrying out a calculation for $M=25$ and by increasing $M$ until no changes were seen in the solution. Case 3 represents an ultraconservative calculation in which more spatial and temporal grid points are employed than needed. Here the parameter $A$, which represents the ratio of the advective term in the material derivative to the time derivative term, is small in all cases, and so linear theory is adequate for treating these flows.

The value of $\partial^{2} \rho / \partial r^{2}$ on the cylinder axis is important in applications, and therefore it is useful to compare our numerical results for this quantity with those calculated previously. Accordingly, the computed values of

$G=10^{9}\left(\partial^{2} \rho / \partial r^{2}\right)_{r=0}$,

as a function of time are shown in fig. 1, along with values for $G$ calculated by Toselli using the theory given in refs. $[1,8]$. As can be seen, the agreement is excellent up to about $t=75 \mu \mathrm{s}$, the time at which the wave reflected from the boundary of the cylinder reaches the axis. The difference between our results and those computed by Toselli can be attributed to the incorrect boundary condition on the radial component of velocity employed in her calculation. In the present study the velocity is taken to vanish at the boundary of the cylinder, as it should, and so the reflected wave is $180^{\circ}$ out of phase with the reflected wave computed by Toselli using the organ pipe boundary condition employed in refs. [1,8]. Similarly, the agreement between our results and Toselli's for the quantity

$H=10^{12}\left(\rho-\rho_{0}\right)_{r=0}$,

shown in fig. 2 is good up to the point when the reflected wave reaches the cylinder axis. The disagreement in values of $G$ in calculating the reflected wave is even more pronounced for cases 2 and 3, and therefore we show

Table 1

Parameters for numerical experiments

\begin{tabular}{lccccrrr}
\hline Parameter & Case 1 & Case 2 & Case 3 & Parameter & Case 1 & Case 2 & Case 3 \\
\hline$p_{0}($ Torr $)$ & 2.05 & 8.05 & 50.05 & $k_{1} \times 10^{4}$ & 11.623 & 2.676 \\
$A \times 10^{6}$ & 3.427 & 2.396 & 2.138 & $k_{2}$ & 4.198 & 12.496 & 62.831 \\
$\gamma$ & 1.640 & 1.655 & 1.659 & $k_{3} \times 10^{4}$ & 16.89 & 3.901 & 0.689 \\
& & & & $k_{4} \times 10^{4}$ & 18.594 & 4.347 & 0.770 \\
$M$ & 30 & 30 & 50 & $k_{5} \times 10^{4}$ & -3.547 & -1.178 & -0.234 \\
TOL $\times 10^{4}$ & 1.0 & 1.0 & 0.1 & & & \\
\hline
\end{tabular}




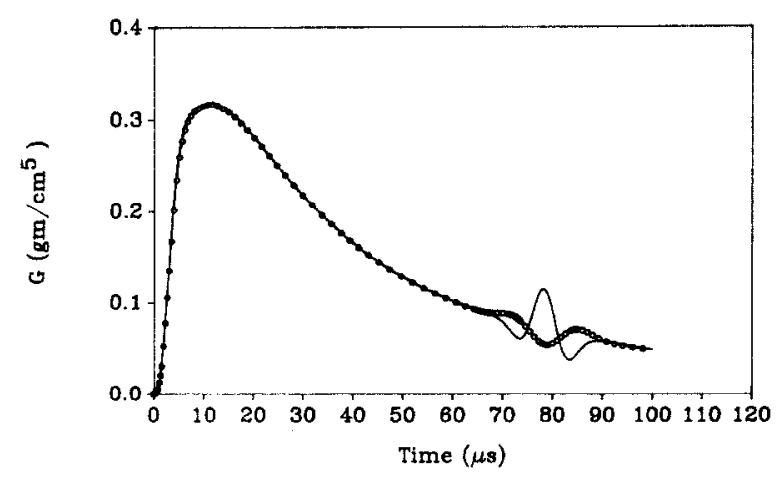

Fig. $1 . G$ in $\mathrm{g} / \mathrm{cm}^{5}$ as a function of time in $\mu$ s for case $1 .(0)$ present theory; (-) theory of Barker and co-workers.

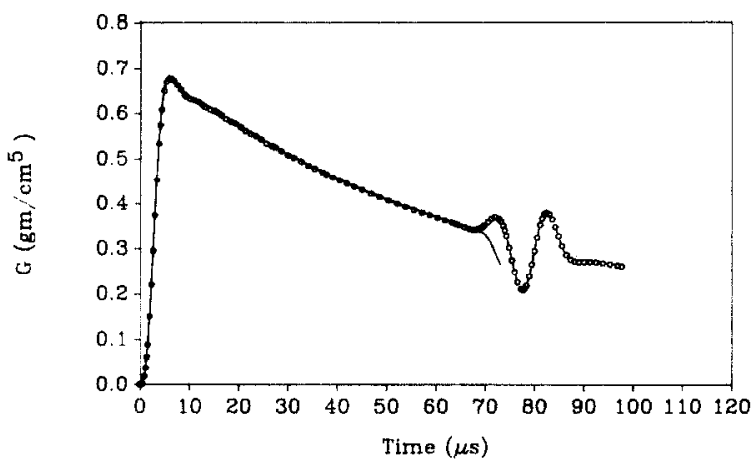

Fig. 3. $G$ in $\mathrm{g} / \mathrm{cm}^{5}$ as a function of time in $\mu$ s for case 2 . (O) present theory; (-) theory of Barker and co-workers.

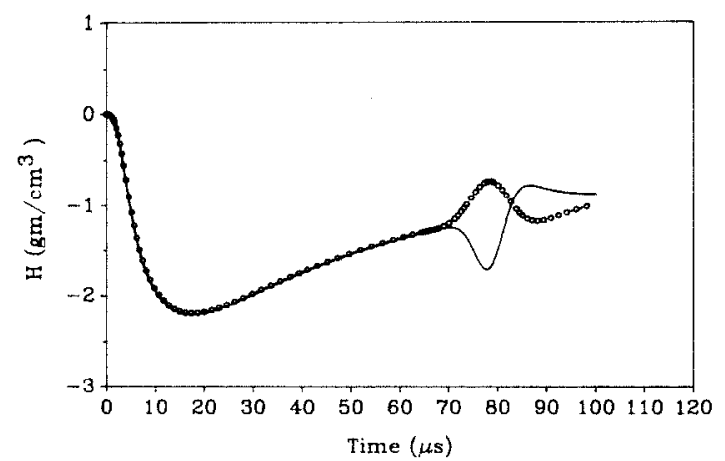

Fig. 2. $H$ in $\mathrm{g} / \mathrm{cm}^{3}$ as a function of time in $\mu$ s for case $1 .(O)$ present theory; (- - - ) theory of Barker and co-workers.

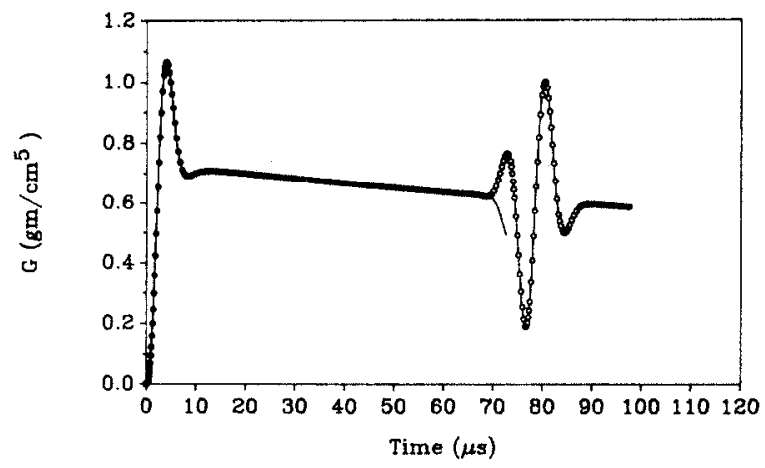

Fig. 4. $G$ in $\mathrm{g} / \mathrm{cm}^{5}$ as a function of time in $\mu$ s for case 3. (O) present theory; $(-)$ theory of Barker and co-workers.

Toselli's numerical results in figs. 3 and 4 only for the period of close agreement between our theories. In this interval the agreement between our theory and the method used in refs. [1,8] is exceptionally good.

Concerning the criticism of the theory of Barker and co-workers in section 1, it can be seen from the numerical results that the neglect of viscous effects in their work is apparently not serious, at least for the parameter values of table 1. Also, the incorrect treatment of the boundary condition on the velocity in Barker's theory does not have a significant effect on the accuracy of the theoretical solution until the return of the reflected wave from the boundary of the cylinder. Therefore, if nonlinear effects are unimportant and if the rate parameter $k_{2}$ is constant, a revised version of the theory of Barker and co-workers in which the correct boundary condition on the velocity is satisfied would probably be adequate in every respect. Since this theory is much less costly in a computational sense than the procedure developed above, it should be used in preference to DIFCOLL or DIFCHEB whenever possible. For treating other cases, the present theory or some comparable numerical treatment is needed.

\section{Concluding remarks}

In refs. $[1,8]$ Barker and co-workers present a detailed comparison between earlier treatments and calculations based on their theory and a discussion on the various effects involved in the competition between energy 
transfer and diffusion. Consequently, since our results and those obtained by Barker and co-workers are virtually identical up to the arrival time of the reflected wave, a comparison of our calculation with previous theories is unnecessary. It remains to point out that the present study is useful in three respects: (i) in assessing the accuracy of earlier theories, as in the comparison of our results with those of Barker and co-workers given above, (ii) in providing a method for treating flows involving a time-dependent rate parameter, and (iii) in possible calculations involving strongly nonlinear flows. The latter application is particularly intriguing in view of the experimental interest in nonlinear flows and their intractibility in terms of analytical treatment.

From a numerical analysis point of view, the novelty of the present study lies in its combination of two accurate numerical procedures, the Chebyshev methods of refs. [11,12] for treating the spatial dependence of the solution, and the Wanner-Rosenbrock methods of refs. $[13,14]$ for advancing the solution in time. In particular, it should be noted that the time-stepping method has a particularly useful aspect, its non-iterative nature, which avoids the excessive computing time required in implicit schemes when many iterations are needed to converge to the solution at a new time step. This feature is extremely useful in possible extensions of the theory to the treatment of nonlinear flows.

\section{Acknowledgement}

The author wishes to thank J.R. Barker and B.M. Toselli for helpful discussions and other assistance.

\section{References}

[1 ] J.R. Barker and B.M. Toselli, in: Photothermal investigations of solids and fluids, ed. J.A. Sell (Academic Press, New York), to be published.

[2] F.R. Grabiner, D.R. Siebert and G.W. Flynn, Chem. Phys. Letters 17 (1972) 189.

[3] N. Presser, J.R. Barker and R.J. Gordon, J. Chem. Phys. 78 (1983) 2163.

[4] J.R. Guckert and R.W. Carr, J. Phys. Chem. 90 (1986) 4286.

[5] D.R. Siebert, Ph.D. Dissertation, Columbia University, New York (1973).

[6] R.T. Bailey, F.R. Cruickshank, R. Guthrie, D. Pugh and I.J.M. Weir, Mol. Phys. 48 (1983) 81.

[7] R.T. Bailey, F.R. Cruickshank, D. Pugh and W. Johnstone, J. Chem. Soc. Faraday II 76 (1980) 633.

[8] J.R. Barker and T. Rothem, Chem. Phys. 68 (1982) 331.

[9] L.D. Landau and E.M. Lifshitz, Fluid mechanics (Addison-Wesley, Reading, MA, 1959).

[10] J.O. Hirschfelder, C.F. Curtiss and R.B. Bird, Molecular theory of gases and liquids (Wiley, New York, 1964).

[11] D. Gottlieb and S.A. Orszag, Numerical Analysis of Spectral Methods: Theory and Applications, in: CBMS Regional Conference Series in Applied Mathematics 26 (Society for Industrial and Applied Mathematics, Philadelphia, 1977).

[12] D. Gottlieb, M.Y. Hussaini and S.A. Orszag, Theory and Applications of Spectral Methods, in: CBMS Proceedings of the Symposium on Spectral Methods (Society for Industrial and Applied Mathematics, Philadelphia, 1984).

[13] L.F. Shampine, ACM Trans. Math. Software 8 (1982) 93.

[14] B.A. Gottwald and G. Wanner, Computing 26 (1981) 355. 